\title{
Differential Game for a Class of Warfare Dynamic Systems with Reinforcement Based on Lanchester Equation
}

\author{
Xiangyong Chen and Jianlong Qiu \\ School of Sciences, Linyi University, Linyi, Shandong 276005, China \\ Correspondence should be addressed to Jianlong Qiu; qiujianlong@lyu.edu.cn \\ Received 19 January 2014; Revised 22 March 2014; Accepted 28 March 2014; Published 22 April 2014 \\ Academic Editor: Ryan Loxton \\ Copyright (C) 2014 X. Chen and J. Qiu. This is an open access article distributed under the Creative Commons Attribution License, \\ which permits unrestricted use, distribution, and reproduction in any medium, provided the original work is properly cited.

\begin{abstract}
This paper concerns the optimal reinforcement game problem between two opposing forces in military conflicts. With some moderate assumptions, we employ Lanchester equation and differential game theory to develop a corresponding optimization game model. After that, we establish the optimum condition for the differential game problem and give an algorithm to obtain the optimal reinforcement strategies. Furthermore, we also discuss the convergence of the algorithm. Finally, a numerical example illustrates the effectiveness of the presented optimal schemes. Our proposed results provide a theoretical guide for both making warfare command decision and assessing military actions.
\end{abstract}

\section{Introduction}

The reinforcement problem in military conflicts is a complex issue on military system science and engineering [1]. It plays a key role in the decision-making tactical of the longtime battles and multiple battle field. This is verified in the analysis of the capture of Iwo Jima [2]. Now, the design of the reinforcement schemes has become a very interesting topic since it is important for drawing up and evaluating warfare plans and decision-making scheme of military actions.

In 1954, Engel firstly presented a generalized Lanchester model with the reinforcement rates [3], which was efficiently applied to analyze the battle of Iwo Jima. Since then, lots of researchers focused on using Lanchester equation [4-11] to solve this kind of inherent problem for determining the optimal reinforcement schemes. Helmbold [12] discussed the direct and inverse solutions of the Lanchester square law equations with general reinforcement schedules. His works provided reliable mathematic basis for the design of the optimal reinforcement schemes of military actions. Sha and Zeng [13] and Zeng and Sha [14] presented the basic frame and a series of key technologies to solve the optimal reinforcement problem in multiple battle field based on optimal control theory and Lanchester equation; their work validates the reasonability and adaptability of the optimal reinforcement problem. Chen $[15,16]$ investigated the optimal control problem for the Lanchester model with the reinforcement by utilizing the iterative regularization method. Chen et al. $[17,18]$ studied the optimal reinforcement problem for winning in military conflicts based on Lanchetser equation and nonlinear optimization technology; their work provides the basis of solving the optimal reinforcement problem. It is easy to note that Lanchester equation has become a powerful quantitative analysis tool to cope with the reinforcement problem.

However, most of work mainly discusses the optimal reinforcement problem of the unilateral decision maker and analyzes the influence of the reinforcement for the outcome of the battle. Very little effort has been devoted to determine the optimal reinforcement of two opposing sides in military conflicts for such dynamic game problem $[19,20]$. Since battle process is a countermeasure process between two belligerent parties, it is necessary to investigate the game problem of warfare dynamic systems with reinforcement.

Li et al. [21, 22] carried out a study on the optimal reinforcement of the two fighting parties. They presented a support differential game optimization model and gave the solution of this game problem. However, they mainly discussed the case that the attrition coefficients are same. The authors $[23,24]$ discussed the reinforcement game problem 
that the attrition coefficients are different. However, they did not consider the influence of the reinforcement rates on both sides, and the objective function they used was relatively simple.

Motivated by the aforementioned discussions, the main aim of this paper is to investigate the optimal reinforcement problem by constructing a corresponding differential game model based on Lanchester equation. We mainly focus on analyzing the optimum conditions and designing a solving algorithm. Meanwhile, the convergence analysis for the algorithm is discussed.

The rest of this paper is organized as follows. In Section 2, the differential game model in determining the optimal reinforcement is presented with some moderate assumptions. In Section 3, the optimum conditions are established, and an algorithm is developed to obtain the optimal reinforcement strategies. Furthermore, the convergence of this method is also discussed. In Section 4, an example is provided to illustrate our theoretical results. Finally, Section 5 presents some concluding remarks.

\section{Description of the Game Model with the Reinforcement}

In this section, we consider the warfare dynamic system model with the reinforcement rates that is described by

$$
\begin{aligned}
& \dot{x}(t)=-\alpha y(t)+u(t), \\
& \dot{y}(t)=-\beta x(t)+v(t), \\
& u\left(t_{0}\right)=v\left(t_{0}\right)=0 ; \quad x(0)=x_{0} ; \quad y(0)=y_{0},
\end{aligned}
$$

where $x(t)$ and $y(t)$ are the strengths of two opposing forces surviving at time $t$ in conflict, $\alpha(>0)$ and $\beta(>0)$ are the constant attrition coefficients that reflect the effectiveness of forces in the unit time, and $u(t)(>0)$ and $v(t)(>0)$ are the reinforcement rates.

We associate (1) with the following objective function:

$$
J(u, v)=x(T)-y(T)+\int_{0}^{T} v(t)-u(t) d t
$$

where $x(T)$ and $y(T)$ are the residual of strengths on both sides in the terminal time $T$.

Now, we consider a military conflict between two opposing forces, and let $X$ be the attacking side, and let $Y$ be the defending side. In the game, $X$ selects the optimal strategy $u^{*}(t)$ to maximize the objective function $J(u, v)$, and $Y$ selects the optimal strategy $v^{*}(t)$ to minimize $J(u, v)$. That is, if there exist $u^{*}(t)$ and $v^{*}(t)$ such that, for any $u(t)$ and $v(t)$, the values of the objective function (2) satisfy

$$
J\left(u, v^{*}\right) \leq J\left(u^{*}, v^{*}\right) \leq J\left(u^{*}, v\right),
$$

then $u^{*}(t)$ and $v^{*}(t)$ are the optimal strategies of differential game.

Moreover, we present some assumptions which will be used in this paper.
$\left(\mathrm{A}_{1}\right)$ The reinforcement rates are $u(t) \in\left[u_{1}, u_{2}\right]$ and $v(t) \epsilon$ $\left[v_{1}, v_{2}\right]$, where $u_{i}(i=1,2)$ and $v_{j}(j=1,2)$ are constants.

$\left(\mathrm{A}_{2}\right)$ Denote $M_{0}$ and $N_{0}$ to be the total amount of the reinforcements of two opposing sides. And the reinforcement from the initial time to the terminal time $T$ satisfies

$$
\int_{0}^{T} u(t) d t \leq M_{0}, \quad \int_{0}^{T} v(t) d t \leq N_{0} .
$$

$\left(\mathrm{A}_{3}\right)$ The reinforcement rates cannot keep the maximum values $u_{2}$ and $v_{2}$ during the whole battle process; that is, the following conditions are satisfied:

$$
\int_{0}^{T} u_{2} d t \geq M_{0}, \quad \int_{0}^{T} v_{2} d t \geq N_{0}
$$

Remark 1. The assumption $\left(\mathrm{A}_{2}\right)$ implies that the total amount of the two opposing sides cannot pass the $M_{0}$ and $N_{0}$; that is,

$$
\int_{0}^{T} u^{*} d t \leq M_{0}, \quad \int_{0}^{T} v^{*} d t \leq N_{0}
$$

hold. The assumption $\left(A_{3}\right)$ represents the limit of the force strengths complementary on both sides. If there is not the condition $\left(\mathrm{A}_{3}\right)$, the optimal strategies of game problem are $u_{2}$ and $v_{2}$, and the game problem in this paper is insignificant.

After giving the above auxiliary statements, we are at the point to investigate the condition for the existence of the optimal reinforcement strategies and develop a procedure of designing the optimal strategies for differential game.

\section{Optimum Condition and the Solutions of the Game Problem}

Considering the existence of the integral inequalities (4) and (5), we cannot use the classic solving theory of differential game to solve the reinforcement game problem. Motivated by $[25,26]$, a new solving theory and algorithm for this reinforcement game problem should be investigated.

We present the solutions of warfare system (1) as follows:

$$
\begin{aligned}
x(t)= & \frac{1}{2}\left(x_{0}-\frac{\sqrt{\alpha \beta}}{\beta} y_{0}\right) e^{\sqrt{\alpha \beta} t}+\frac{1}{2}\left(x_{0}+\frac{\sqrt{\alpha \beta}}{\beta} y_{0}\right) e^{-\sqrt{\alpha \beta} t} \\
+ & \frac{1}{2} \int_{0}^{t}\left(\left(u-\frac{\sqrt{\alpha \beta}}{\beta} v\right) e^{\sqrt{\alpha \beta(} t-\tau)}\right. \\
& \left.+\left(u+\frac{\sqrt{\alpha \beta}}{\beta} v\right) e^{-\sqrt{\alpha \beta(} t-\tau)}\right) d \tau,
\end{aligned}
$$




$$
\begin{aligned}
y(t)= & \frac{1}{2}\left(y_{0}-\frac{\sqrt{\alpha \beta}}{\alpha} x_{0}\right) e^{\sqrt{\alpha \beta} t}+\frac{1}{2}\left(y_{0}+\frac{\sqrt{\alpha \beta}}{\alpha} x_{0}\right) e^{-\sqrt{\alpha \beta} t} \\
+ & \frac{1}{2} \int_{0}^{t}\left(\left(v-\frac{\sqrt{\alpha \beta}}{\alpha} u\right) e^{\sqrt{\alpha \beta}(t-\tau)}\right. \\
& \left.+\left(v+\frac{\sqrt{\alpha \beta}}{\alpha} u\right) e^{-\sqrt{\alpha \beta}(t-\tau)}\right) d \tau .
\end{aligned}
$$

Then, the objective function $J(u, v)$ is

$$
J(u, v)=\Delta_{1}+\int_{0}^{T}\left(\left(\Delta_{2}-1\right) u-\left(\Delta_{3}-1\right) v\right) d t,
$$

where

$$
\begin{aligned}
\Delta_{1}= & \frac{1}{2}\left(x_{0}-y_{0}+\frac{\sqrt{\alpha \beta}}{\alpha} x_{0}-\frac{\sqrt{\alpha \beta}}{\beta} y_{0}\right) e^{\sqrt{\alpha \beta} T} \\
& +\frac{1}{2}\left(x_{0}-y_{0}-\frac{\sqrt{\alpha \beta}}{\alpha} x_{0}+\frac{\sqrt{\alpha \beta}}{\beta} y_{0}\right) e^{-\sqrt{\alpha \beta} T}, \\
\Delta_{2}= & \frac{1}{2}\left(\left(1+\frac{\sqrt{\alpha \beta}}{\alpha}\right) e^{\sqrt{\alpha \beta(T-t)}}+\left(1-\frac{\sqrt{\alpha \beta}}{\alpha}\right) e^{-\sqrt{\alpha \beta(T-t)}}\right), \\
\Delta_{3}= & \frac{1}{2}\left(\left(1+\frac{\sqrt{\alpha \beta}}{\beta}\right) e^{\sqrt{\alpha \beta(}(T-t)}+\left(1-\frac{\sqrt{\alpha \beta}}{\beta}\right) e^{-\sqrt{\alpha \beta(T-t)}}\right) .
\end{aligned}
$$

Denote

$$
f(u, v)=\int_{0}^{T}\left(\left(\Delta_{2}-1\right) u-\left(\Delta_{3}-1\right) v\right) d t,
$$

and then with (7) and the fact that $x_{0}, y_{0}$, and $T$ are the known constants, we declare that if there exist $u^{*}(t)$ and $v^{*}(t)$ such that

$$
f\left(u, v^{*}\right) \leq f\left(u^{*}, v^{*}\right) \leq f\left(u^{*}, v\right),
$$

then (3) is satisfied, and $u^{*}(t)$ and $v^{*}(t)$ are the optimal strategies of differential game (1) and (2).

By constructing Lagrange function

$$
L(u, v, \lambda, \mu, t)=\left(\Delta_{2}-1\right) u-\left(\Delta_{3}-1\right) v-\lambda u+\mu v,
$$

we obtain the following theorem about the optimum condition for the existence of the optimal reinforcement strategies of differential game.

Theorem 2. If there exist constants $\lambda>0, \mu>0$ and the reinforcement rates $u^{*}(t)$ and $v^{*}(t)$, which satisfy

$$
\int_{0}^{T} u d t \leq \int_{0}^{T} u^{*} d t \leq M_{0}, \quad \int_{0}^{T} v d t \leq \int_{0}^{T} v^{*} d t \leq N_{0},
$$

such that the inequality

$$
L\left(u, v^{*}, \lambda, \mu, t\right) \leq L\left(u^{*}, v^{*}, \lambda, \mu, t\right) \leq L\left(u^{*}, v, \lambda, \mu, t\right)
$$

holds, then the inequalities (3) and (11) hold and $u^{*}(t)$ and $v^{*}(t)$ are the optimal reinforcement strategies of differential game (1) and (2).
Proof. We rewrite (12) as

$$
L(u, v, \lambda, \mu, t)=\left(\Delta_{2}-\lambda-1\right) u-\left(\Delta_{3}-\mu-1\right) v .
$$

With (14) and (15), we have

$$
L\left(u^{*}, v^{*}, \lambda, \mu, t\right)=\max _{u}\left(\left(\Delta_{2}-\lambda-1\right) u-\left(\Delta_{3}-\mu-1\right) v^{*}\right) .
$$

Then, we conclude that the following results about the optimal strategies $u^{*}$ hold.

(1) If $\Delta_{2}>\lambda+1$, the optimal reinforcement rate $u^{*}$ is the upper bound of $u$. Otherwise, we assume that $u_{m}\left(u_{1}<u_{m}<\right.$ $u_{2}$ ) is another optimal strategy and get

$$
\max _{u} L\left(u, v^{*}, \lambda, \mu, t\right)=\left(\Delta_{2}-\lambda-1\right) u_{m}-\left(\Delta_{3}-\mu-1\right) v^{*} .
$$

So

$$
\left(\Delta_{2}-\lambda-1\right) u_{m} \geq\left(\Delta_{2}-\lambda-1\right) u, \quad u \in\left[u_{1}, u_{2}\right] .
$$

However, we know that

$$
\left(\Delta_{2}-\lambda-1\right) u_{m} \leq\left(\Delta_{2}-\lambda-1\right) u_{2} .
$$

It follows that

$$
\max _{u} L\left(u, v^{*}, \lambda, \mu, t\right) \leq\left(\Delta_{2}-\lambda-1\right) u_{2}-\left(\Delta_{3}-\mu-1\right) v^{*} .
$$

From (16) and (20), it is easy to verify that (17) does not hold. That is, when $\Delta_{2}>\lambda+1$, we get that $u^{*}=u_{2}$ and

$$
L\left(u^{*}, v^{*}, \lambda, \mu, t\right) \geq L\left(u, v^{*}, \lambda, \mu, t\right) .
$$

(2) Similarly, if $\Delta_{2}<\lambda+1$, the optimal reinforcement rate $u^{*}$ is the lower bound of $u$; that is, $u^{*}=u_{1}$ and (21) holds.

On the other hand, for any reinforcement strategy $v$, it follows from (14) that

$$
L\left(u^{*}, v^{*}, \lambda, \mu, t\right)=\min _{v}\left(\left(\Delta_{2}-\lambda-1\right) u^{*}-\left(\Delta_{3}-\mu-1\right) v\right) .
$$

Similarly, we get the following results about the optimal strategies $v^{*}$.

(3) If $\Delta_{3}>\mu+1$, the optimal reinforcement rate $v^{*}$ is the upper bound of $v$. That is, $v^{*}=v_{2}$ and

$$
L\left(u^{*}, v^{*}, \lambda, \mu, t\right) \leq L\left(u^{*}, v, \lambda, \mu, t\right) .
$$

(4) If $\Delta_{3}<\mu+1$, the optimal reinforcement rate $v^{*}$ is the lower bound of $v$. That is, $v^{*}=v_{1}$ and (23) holds.

After preparing the above, we integrate both sides of (21) and (23) from 0 to $T$ and get

$$
\begin{aligned}
\int_{0}^{T} L\left(u, v^{*}, \lambda, \mu, t\right) d t & \leq \int_{0}^{T} L\left(u^{*}, v^{*}, \lambda, \mu, t\right) d t \\
& \leq \int_{0}^{T} L\left(u^{*}, v, \lambda, \mu, t\right) d t
\end{aligned}
$$


From (13), we have

$$
\begin{aligned}
& f\left(u^{*}, v^{*}\right)-f\left(u, v^{*}\right) \geq \lambda \int_{0}^{T} u^{*} d t-\lambda \int_{0}^{T} u d t \geq 0, \\
& f\left(u^{*}, v^{*}\right)-f\left(u^{*}, v\right) \leq \mu \int_{0}^{T} v d t-\mu \int_{0}^{T} v^{*} d t \leq 0 .
\end{aligned}
$$

With the help of the above inequalities, we declare that the inequalities (3) and (11) hold. That is, $u^{*}(t)$ and $v^{*}(t)$ are the optimal reinforcement strategies of differential game (1) and (2). This completes the proof.

For the fact that $L(u, v, \lambda, \mu, t)$ is the separable function, it is not difficult to note that we cannot use the partial derivative technique to obtain the optimal strategies. According to Theorem 2, the optimal strategies $u^{*}(t)$ and $v^{*}(t)$ are obtained as

$$
\begin{aligned}
& u^{*}(t)= \begin{cases}u_{2}, & \Delta_{2} \geq \lambda+1 \\
u_{1}, & \Delta_{2}<\lambda+1,\end{cases} \\
& v^{*}(t)= \begin{cases}v_{2}, & \Delta_{3} \geq \mu+1 \\
v_{1}, & \Delta_{3}<\mu+1 .\end{cases}
\end{aligned}
$$

Let $\Delta_{2}=\lambda+1$ and $\Delta_{3}=\mu+1$; it is easy to get

$$
\begin{aligned}
t_{\lambda}= & T-\ln \left(\frac{\lambda+1+\sqrt{(\lambda+1)^{2}-(1-\beta / \alpha)}}{(1+\sqrt{\alpha \beta} / \alpha)}\right) \\
& \times(\sqrt{\alpha \beta})^{-1}, \\
t_{\mu}= & T-\ln \left(\frac{\mu+1+\sqrt{(\mu+1)^{2}-(1-\alpha / \beta)}}{(1+\sqrt{\alpha \beta} / \beta)}\right) \\
& \times(\sqrt{\alpha \beta})^{-1} .
\end{aligned}
$$

Rewrite (26) as the following form:

$$
\begin{aligned}
& u^{*}(t)= \begin{cases}u_{2}, & t \in\left[0, t_{\lambda}\right] \\
u_{1}, & t \in\left(t_{\lambda}, T\right],\end{cases} \\
& v^{*}(t)= \begin{cases}v_{2}, & t \in\left[0, t_{\mu}\right] \\
v_{1}, & t \in\left(t_{\mu}, T\right] .\end{cases}
\end{aligned}
$$

Remark 3. The tactical significance of (28) is that $X$ employs the maximum reinforcement rate to support the troop if $0<t<t_{\lambda}$. Otherwise, the minimum reinforcement rate is employed. In addition, $Y$ employs the maximum reinforcement rate to support the troop if $0<t<t_{\mu}$ and employs the minimum reinforcement rate $v_{1}$ if $t_{\mu}<t<T$.

Since the parameters $\lambda$ and $\mu$ are unknown, we note that $t_{\lambda}$ and $t_{\mu}$ cannot be directly calculated and the optimal strategies $u^{*}(t)$ and $v^{*}(t)$ cannot be directly determined. Then, an obvious optimization algorithm for determining the optimal reinforcement strategies is presented as follows.
Step 1. Set $k=0$ and $\widehat{k}=0$ and choose the initial values $\lambda_{0}>0$ and $\mu_{0}>0$ such that

$$
\left(\lambda_{0}+1\right)^{2}-\left(1-\frac{\beta}{\alpha}\right) \geq 0, \quad\left(\mu_{0}+1\right)^{2}-\left(1-\frac{\alpha}{\beta}\right) \geq 0 .
$$

Step 2. From (26) and (28), we get $t_{\lambda_{k}}, t_{\mu_{\widehat{k}}}, u_{\lambda_{k}}^{*}, v_{\mu_{\hat{k}}}^{*}$, and

$$
\begin{aligned}
& \int_{0}^{T} u_{\lambda_{k}}^{*} d t=u_{2} t_{\lambda_{k}}+u_{1}\left(T-t_{\lambda_{k}}\right), \\
& \int_{0}^{T} v_{\mu_{\hat{k}}}^{*} d t=v_{2} t_{\mu_{\hat{k}}}+v_{1}\left(T-t_{\mu_{\hat{k}}}\right) .
\end{aligned}
$$

Step 3. Set the confidence intervals of $t_{\lambda}$ and $t_{\mu}$ to be

$$
\left|t_{\lambda}^{*}-t_{\lambda}\right| \leq \epsilon_{1}, \quad\left|t_{\mu}^{*}-t_{\mu}\right| \leq \epsilon_{2} .
$$

Thus, the calculation errors of the total of the reinforcements satisfy

$$
\left|\int_{0}^{T} u^{*} d t-M_{0}\right| \leq \omega_{1}, \quad\left|\int_{0}^{T} v^{*} d t-N_{0}\right| \leq \omega_{2} .
$$

If

$$
M_{0}-\omega_{1} \leq \int_{0}^{T} u^{*} d t \leq M_{0}, \quad N_{0}-\omega_{2} \leq \int_{0}^{T} v^{*} d t \leq N_{0}
$$

holds, then $u_{\lambda_{k}}^{*}$ and $v_{\mu_{\hat{k}}}^{*}$ are the optimal reinforcement strategies; else go to Step 4.

Step 4. Adjust the new values $\lambda_{k+1}>0$ and $\mu_{\hat{k}+1}>0$, set $k=k+1$ and $\widehat{k}=\widehat{k}+1$, then $\lambda_{k+1}=\lambda_{k}+\eta$ and $\mu_{\widehat{k}+1}=\mu_{\widehat{k}}+\eta$, where $\eta(0<\eta<1)$ is the step length, and go back to Step 2 .

Remark 4. Using the above algorithm, we obtain a feasible solution of the reinforcement problem. From the fact that the calculation cost depends largely on the initial values set, we know that if we select initial values $\lambda_{0}, \mu_{0}, \epsilon_{1}, \epsilon_{2}, \omega_{1}$, and $\omega_{2}$ properly, the calculation cost can be decreased and more accurate solution can be obtained.

In the rest of this section, we analyze the convergence of the above algorithm. According to $\left(\mathrm{A}_{2}\right),(31)$, and (32), we know that the desired optimal reinforcement rates $u^{*}$ and $v^{*}$ satisfy

$$
\int_{0}^{T} u^{*} d t=M_{0}, \quad \int_{0}^{T} v^{*} d t=N_{0} .
$$

So, the desired values $t_{\lambda}^{*}$ and $t_{\mu}^{*}$ are

$$
t_{\lambda}^{*}=\frac{M_{0}-u_{1} T}{u_{2}-u_{1}}, \quad t_{\mu}^{*}=\frac{N_{0}-v_{1} T}{v_{2}-v_{1}} .
$$

Meanwhile, the sequence of functions $\left\{\int_{0}^{T} u_{\lambda_{k}} d t\right\}_{k}$ and $\left\{\int_{0}^{T} v_{\mu_{\hat{k}}} d t\right\}_{\widehat{k}}$ is obtained based on the above $k$ steps computation process.

Next, we prove that $\left\{\int_{0}^{T} u_{\lambda_{k}} d t\right\}_{k}$ and $\left\{\int_{0}^{T} v_{\mu_{\widehat{k}}} d t\right\}_{\widehat{k}}$ converge to the desired values $M_{0}$ and $N_{0}$ in finite steps, respectively. 
Theorem 5. Choose an initial value $\lambda_{0}>0$ and the step length $\eta(0<\eta<1)$; the sequence of function $\left\{\int_{0}^{T} u_{\lambda_{k}} d t\right\}_{k}$ is convergent to the limit value $M_{0}$ after finite steps.

Proof. It is easy to get

$$
\begin{aligned}
& \left|\int_{0}^{T} u_{\lambda_{k}} d t-M_{0}\right| \\
& =\left|u_{2} t_{\lambda_{k}}+u_{1}\left(T-t_{\lambda_{k}}\right)-M_{0}\right| \\
& =\frac{u_{2}-u_{1}}{\sqrt{\alpha \beta}} \\
& \left.\quad \times \mid \frac{u_{2} T-M_{0}}{u_{2}-u_{1}}\right) \\
& \quad-\ln \left(\frac{\lambda_{k}+1+\sqrt{\left(\lambda_{k}+1\right)^{2}-(1-\beta / \alpha)}}{(1+\sqrt{\alpha \beta} / \alpha)}\right) .
\end{aligned}
$$

Setting $\lambda_{k}=\lambda_{0}+k \eta$, we rewrite (36) as

$$
\begin{aligned}
& \left|\int_{0}^{T} u_{\lambda_{k}} d t-M_{0}\right| \\
& =\frac{u_{2}-u_{1}}{\sqrt{\alpha \beta}} \\
& \quad \times \mid \frac{u_{2} T-M_{0}}{u_{2}-u_{1}} \\
& \quad-\ln \left(\frac{\lambda_{0}+k \eta+1+\sqrt{\left(\lambda_{0}+k \eta+1\right)^{2}-(1-\beta / \alpha)}}{(1+\sqrt{\alpha \beta} / \alpha)}\right) .
\end{aligned}
$$

For all $\varepsilon>0$ and the step times $k\left(k \in Z^{*}\right)$ satisfies

$$
\begin{aligned}
& \frac{C^{2} e^{2 D}-2\left(\lambda_{0}+1\right) C e^{D}+(1-\beta / \alpha)}{2 \eta C e^{D}} \\
& <k<\frac{C^{2} e^{2 E}-2\left(\lambda_{0}+1\right) C e^{E}+(1-\beta / \alpha)}{2 \eta C e^{E}},
\end{aligned}
$$

we have

$$
\left|\int_{0}^{T} u_{\lambda_{k}} d t-M_{0}\right|<\varepsilon
$$

where $C=1+\sqrt{\alpha \beta} / \alpha, D=\sqrt{\alpha \beta}\left(u_{2} T-M_{0}-\varepsilon\right) /\left(u_{2}-u_{1}\right)$, and $E=\sqrt{\alpha \beta}\left(u_{2} T-M_{0}+\varepsilon\right) /\left(u_{2}-u_{1}\right)$. That is, $\left\{\int_{0}^{T} u_{\lambda_{k}} d t\right\}_{k}$ converges to the limit value $M_{0}$ after $k$ steps.

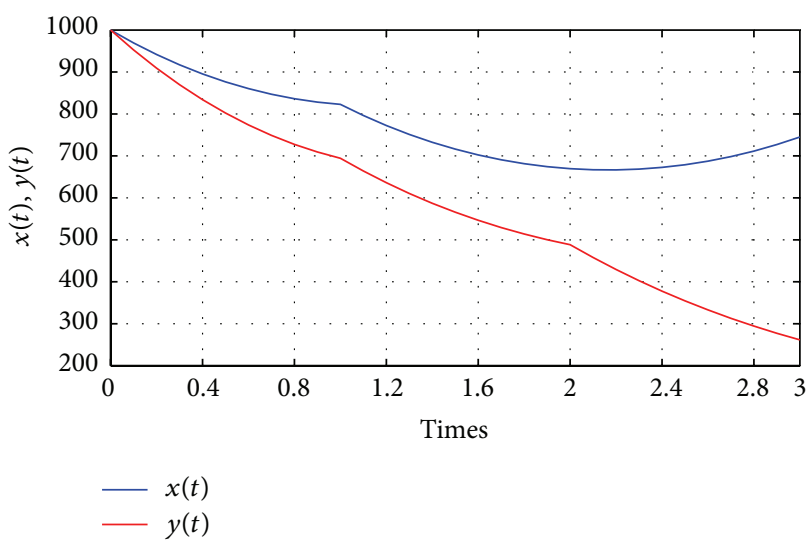

Figure 1: Time behavior for state variables $x(t)$ and $y(t)$ with the optimal strategies $u^{*}, v^{*}$.

Remark 6. We note that the step number $k$ in Theorem 5 is not given explicitly, and it should be selected as follows:

$$
k=\left\{\begin{array}{l}
\left\lfloor\frac{C^{2} e^{2 E}-2\left(\lambda_{0}+1\right) C e^{E}+(1-\beta / \alpha)}{2 \eta C e^{E}}\right\rfloor+1, \\
\left.\qquad \frac{C^{2} e^{2 E}-2\left(\lambda_{0}+1\right) C e^{E}+(1-\beta / \alpha)}{2 \eta C e^{E}}\right\rfloor, \\
\text { otherwise, }
\end{array}\right.
$$

where

$$
\begin{aligned}
S= & \left\lfloor\frac{C^{2} e^{2 E}-2\left(\lambda_{0}+1\right) C e^{E}+(1-\beta / \alpha)}{2 \eta C e^{E}}\right. \\
& \left.-\frac{C^{2} e^{2 D}-2\left(\lambda_{0}+1\right) C e^{D}+(1-\beta / \alpha)}{2 \eta C e^{D}}\right\rfloor .
\end{aligned}
$$

Theorem 7. Choose an initial value $\mu_{0}$ and the step length $\theta(0<\theta<1)$; the sequence of function $\left\{\int_{0}^{T} \nu_{\mu_{\hat{k}}} d t\right\}_{\widehat{k}}$ converges to the limit value $N_{0}$ after finite steps.

Proof. Since the proof is similar to Theorem 5, we omit it.

Remark 8. In Theorem $7, \widehat{k}$ is selected as

$$
\widehat{k}=\left\{\begin{array}{l}
\left\lfloor\frac{F^{2} e^{2 H}-2\left(\mu_{0}+1\right) F e^{H}+(1-\alpha / \beta)}{2 \theta F e^{E}}\right\rfloor+1, \\
S_{S^{\prime}=0}=0 \\
\left\lfloor\frac{F^{2} e^{2 H}-2\left(\mu_{0}+1\right) F e^{H}+(1-\alpha / \beta)}{2 \theta F e^{E}}\right\rfloor, \\
\text { other case, }
\end{array}\right.
$$




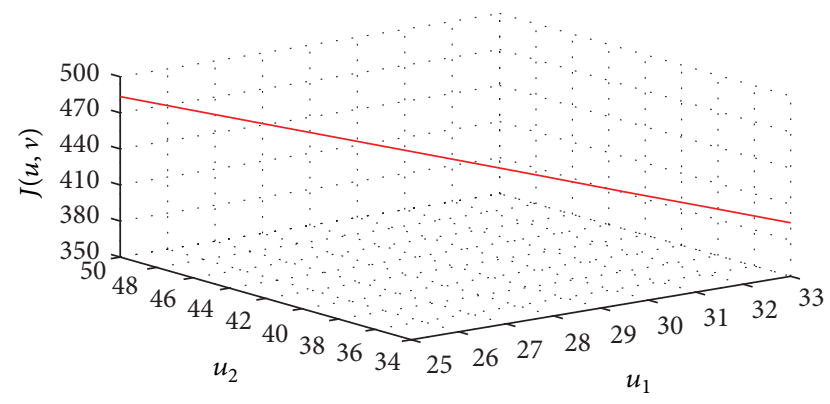

FIGURE 2: Changes of $J(u, v)$ with the different reinforcement rate $u$ for $X$.

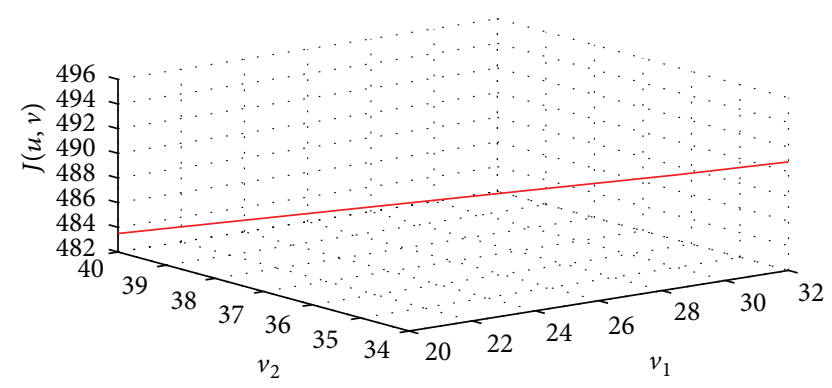

FIGURE 3: Changes of $J(u, v)$ with the different reinforcement rate $v$ for $Y$.

where

$$
\begin{gathered}
F=1+\sqrt{\alpha \beta}, \quad G=\frac{\sqrt{\alpha \beta}\left(v_{2} T-N_{0}-\varepsilon\right)}{v_{2}-v_{1}}, \\
H=\frac{\sqrt{\alpha \beta}\left(v_{2} T-N_{0}+\varepsilon\right)}{v_{2}-v_{1}}, \\
S^{\prime}=\left\lfloor\frac{F^{2} e^{2 H}-2\left(\mu_{0}+1\right) F e^{H}+(1-\alpha / \beta)}{2 \theta F e^{E}}\right. \\
\left.-\frac{F^{2} e^{2 G}-2\left(\mu_{0}+1\right) F e^{G}+(1-\alpha / \beta)}{2 \theta C e^{G}}\right\rfloor .
\end{gathered}
$$

\section{Numerical Example}

Since Lanchester equation is a powerful tool for analyzing real wars quantitatively and determining tactics in combat simulations, it produces reasonably good predictions. We confirm that our presented game model based on Lanchester equation is very useful to copy with some specific practical military problems when the corresponding parameter values in military conflicts are obtained.

Thus, in this section, we present a numerical example to illustrate the effectiveness of our theoretical results. The initial force strengths and the total reinforcements are

$$
\begin{aligned}
& x_{0}=1000, \quad y_{0}=1000, \quad \alpha=0.4, \\
& \beta=0.9, \quad M_{0}=100, \quad N_{0}=100 \text {, }
\end{aligned}
$$

TABLE 1: The feasible solutions of the game problem when setting the different values of $T, M_{0}$ and $N_{0}$ with $\alpha=0.4, \beta=0.9$.

\begin{tabular}{ccccccc}
\hline$T$ & $t_{\lambda}$ & $t_{\mu}$ & $\int_{0}^{T} u^{*} d t$ & $\int_{0}^{T} v^{*} d t$ & $M_{0}$ & $N_{0}$ \\
\hline 3 & 0.9842 & 1.9790 & 99.6059 & 99.5802 & 100 & 100 \\
4 & 3.1714 & 3.4923 & 179.2849 & 149.8457 & 180 & 150 \\
5 & 2.9976 & 2.4986 & 199.9394 & 149.9723 & 200 & 150 \\
10 & 5.9857 & 7.4986 & 399.6282 & 349.9723 & 400 & 350 \\
\hline
\end{tabular}

TABLE 2: The feasible solutions of the game problem when setting the different values of $\alpha$ and $\beta$ change with $T=3, M_{0}=N_{0}=100$.

\begin{tabular}{cccccc}
\hline$\alpha$ & $\beta$ & $t_{\lambda}$ & $t_{\mu}$ & $\int_{0}^{T} u^{*} d t$ & $\int_{0}^{T} v^{*} d t$ \\
\hline 0.5 & 0.85 & 0.9793 & 1.9779 & 99.4821 & 99.5589 \\
0.6 & 0.9 & 0.9915 & 1.9940 & 99.7883 & 99.8803 \\
0.8 & 0.9 & 0.9912 & 1.9771 & 99.7801 & 99.5413 \\
0.55 & 0.75 & 0.9775 & 1.9839 & 99.4384 & 99.6778 \\
0.35 & 0.75 & 0.9841 & 1.9583 & 99.6019 & 99.1657 \\
\hline
\end{tabular}

and the battle terminal time is $T=3$. From $\left(\mathrm{A}_{1}\right)$, we chose that $u_{1}=25, u_{2}=50, v_{1}=20$, and $v_{2}=40$. In the proposed solving algorithm, we set the initial values $\lambda_{0}=0$ and $\mu_{0}=0$ and the step length $\eta=0.01$. Let the confidence intervals of $t_{\lambda}, t_{\mu}$ and the calculation errors of the total reinforcements satisfy

$$
\begin{aligned}
& \left|t_{\lambda}^{*}-t_{\lambda}\right| \leq 0.1, \quad\left|t_{\mu}^{*}-t_{\mu}\right| \leq 0.1, \\
& \left|\int_{0}^{T} u^{*} d t-M_{0}\right| \leq 0.9, \quad\left|\int_{0}^{T} v^{*} d t-N_{0}\right| \leq 0.9 .
\end{aligned}
$$

Solving the differential game problem by MATLAB Toolbox yields the feasible solutions as $\lambda=3.1150, \mu=0.6280$, $t_{\lambda}=0.9842$, and $t_{\mu}=1.9790$. From (28), we get the optimal reinforcement rates $u^{*}$ and $v^{*}$ as

$$
\begin{aligned}
& u^{*}(t)= \begin{cases}u_{2}, & t \in[0,0.9842] \\
u_{1}, & t \in(0.9842, T]\end{cases} \\
& v^{*}(t)= \begin{cases}v_{2}, & t \in[0,1.9790] \\
v_{1}, & t \in(1.9790, T]\end{cases}
\end{aligned}
$$

The total reinforcement on both sides is

$$
\int_{0}^{T} u^{*} d t=99.6059, \quad \int_{0}^{T} v^{*} d t=99.5802 .
$$

Furthermore, we get the optimal object function value $J=$ 483.46.

Figure 1 shows the time behavior for state variables of warfare dynamic system with the optimal strategies $u^{*}$ and $v^{*}$. We note that the state values $x(t)$ and $y(t)$ changed at $t=0.9842$ and $t=1.9790$. Figures 2 and 3 show the changes of $J(u, v)$ with the different $u$ and $v$. It is clear that (3) holds, which implies that (46) is the optimal reinforcement strategies of the game problem in this example. 
We also analyze the feasible solutions $t_{\lambda}, t_{\mu}, \int_{0}^{T} u^{*} d t$, and $\int_{0}^{T} v^{*} d t$ by choosing the different parameters $T, M_{0}, N_{0}$, $\alpha$, and $\beta$. Table 1 gives the feasible solutions of the game problem when setting the different values of $T, M_{0}$, and $N_{0}$ and keeping $\alpha=0.4$ and $\beta=0.9$ fixed. Table 2 demonstrates the computation results when $\alpha$ and $\beta$ change with, $T=3$, $M_{0}=N_{0}=100$. It can be determined from Tables 1 and 2 that when (45) is satisfied, the solving algorithm proposed in this paper is practicable and valid.

\section{Conclusions}

This paper discusses a differential game problem of warfare dynamic system with the reinforcement. An optimization game model is established based on Lanchester equation and differential game theory. Then the optimum condition and the solving method about the game problem are given. Simulation results illustrate the effectiveness of proposed optimal strategies. This is of great significance in analyzing quantitatively military actions. Moreover, employing advanced control techniques $[24,27]$ to investigate warfare command game problem is our future research directions.

\section{Conflict of Interests}

The authors declare that there is no conflict of interests regarding the publication of this paper.

\section{Acknowledgments}

The authors would like to express their deepest gratitude to Dr. Jianwei Zhou for his helpful suggestions on the English writing of the revised version. This work was supported in part by the National Natural Science Foundation of China, under Grants 61273012, 11301252, and 11201212, a Project of Shandong Province Higher Educational Science and Technology Program under Grants J13LI11 and J12LI58, and the Applied Mathematics Enhancement Program (AMEP) of Linyi University.

\section{References}

[1] D. H. Wagner, M. W. Charles, and T. J. Sanders, Naval Operations Analysis, Naval Institute Press, Annapolis, Md, USA, 1999.

[2] R. Andrew, Battle Story: Iwo Jima 1945, The History Press, Stroud, UK, 2012.

[3] J. H. Engel, "A verification of Lanchester's law," Journal of the Operations Research Society of America, vol. 2, no. 2, pp. 163171, 1954.

[4] F. W. Lanchester, Aircraft in Warfare: the Dawn of the Fourth Arm, Constable, London, UK, 1916, and Lanchester Press, 1999.

[5] J. G. Taylor, Lanchester Models of Warfare, Volumes 2: Operations Research Society of America, Military Applications Section, Arlington, Va, USA, 1983.

[6] J. C. Sha, Mathematic Tactics, Science Press, Beijing, China, 2003.

[7] J. G. Taylor, “ Lanchester-type models of warfare and optimal control," Naval Research Logistics, vol. 21, no. 1, pp. 79-106, 1974.
[8] T. Keane, "Combat modelling with partial differential equations," Applied Mathematical Modelling, vol. 35, no. 6, pp. $2723-$ 2735, 2011.

[9] E. González and M. Villena, "Spatial Lanchester models," European Journal of Operational Research, vol. 210, no. 3, pp. 706-715, 2011.

[10] I. R. Johnson and N. J. MacKay, "Lanchester models and the battle of Britain," Naval Research Logistics, vol. 58, no. 3, pp. 210222, 2011.

[11] J. Zhang, C. Xu, L. Gong, and D. Yuan, "The mathematical model based on the battle of Berlin," in Proceedings of the 8th International Conference on Fuzzy Systems and Knowledge Discovery, pp. 2133-2136, IEEE Press, July 2011.

[12] R. L. Helmbold, "Direct and inverse solution of the Lanchester square law with general reinforcement schedules," European Journal of Operational Research, vol. 77, no. 3, pp. 486-495, 1994.

[13] J. C. Sha and A. J. Zeng, "Research on the warfare theory of Lanchester and tactics," in Proceedings of the Control and Decision Conference of China, pp. 1134-1136, Xiamen, China, 1994.

[14] A. J. Zeng and J. C. Sha, "The optimal reinforcement problem in military conficts," in Proceedings of the Control and Decision Conference of China, pp. 1142-1145, Xiamen, China, 1994.

[15] H. Chen, "An optimal control problem in determining the optimal reinforcement schedules for the Lanchester equations," Computers and Operations Research, vol. 30, no. 7, pp. 1051-1066, 2003.

[16] H. Chen, "A non-linear inverse Lanchester square law problem in estimating the force-dependent attrition coefficients," European Journal of Operational Research, vol. 182, no. 2, pp. 911-922, 2007.

[17] X. Chen, Y. Jing, C. Li, and X. Liu, "Optimal strategies for winning in military conflicts based on Lanchester equation," Control and Decision, vol. 26, no. 6, pp. 946-950, 2011.

[18] X. Chen, Y. Jing, C. Li, and M. Li, "Warfare command stratagem analysis for winning based on Lanchester attrition models," Journal of Systems Science and Systems Engineering, vol. 21, no. 1, pp. 94-105, 2012.

[19] R. Isaacs, Differential Games: A Mathematical Theory with Applications to Warfare and Pursuit, Control and Optimization, Wiley, New York Press, 1965.

[20] J. G. Taylor, "Differential-game examination of optimal timesequential fire-support strategies," Naval Research Logistics Quarterly, vol. 25, no. 2, pp. 322-355, 1983.

[21] D. F. Li, A. S. Tan, and F. Luo, "Optimization model of reinforcements based on differential game and its solving method," Operations Research and Management Science, vol. 11, no. 4, pp. 16-20, 2002.

[22] D. F. Li and Q. H. Chen, "Troops support differential game optimization model and solution," Fire Control and Command Control, vol. 29, no. 1, pp. 41-43, 2004.

[23] X. Y. Chen, Y. W. Jing, C. J. Li et al., "Differential game model and its solutions for force resource complementary via Lanchester square law equation," in Proceedings of the 18th IFAC World Congress, pp. 1024-1030, Milano, Italy, 2011.

[24] X. Y. Chen and A. C. Zhang, "Modeling and optimal control of a class of warfare hybrid dynamic systems based on Lanchester $(n, 1)$ attrition model," Mathematical Problems in Engineering, vol. 2014, Article ID 481347, 7 pages, 2014.

[25] Q. Lin, R. Loxton, K. L. Teo, and Y. H. Wu, "A new computational method for a class of free terminal time optimal control 
problems," Pacific Journal of Optimization, vol. 7, no. 1, pp. 6381, 2011.

[26] Q. Lin, R. Loxton, K. L. Teo, and Y. H. Wu, “Optimal control computation for nonlinear systems with state-dependent stopping criteria," Automatica, vol. 48, no. 9, pp. 2116-2129, 2012.

[27] Q. Lin, R. Loxton, and K. L. Teo, "The control parameterization method for nonlinear optimal control: a survey," Journal of Industrial and Management Optimization, vol. 10, no. 1, pp. 275309, 2014. 


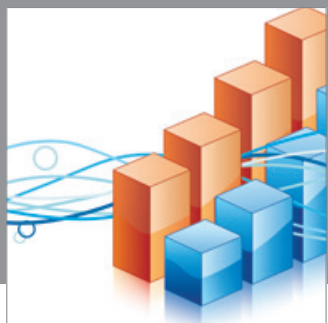

Advances in

Operations Research

mansans

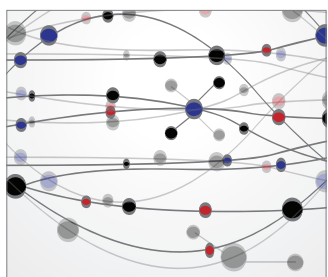

The Scientific World Journal
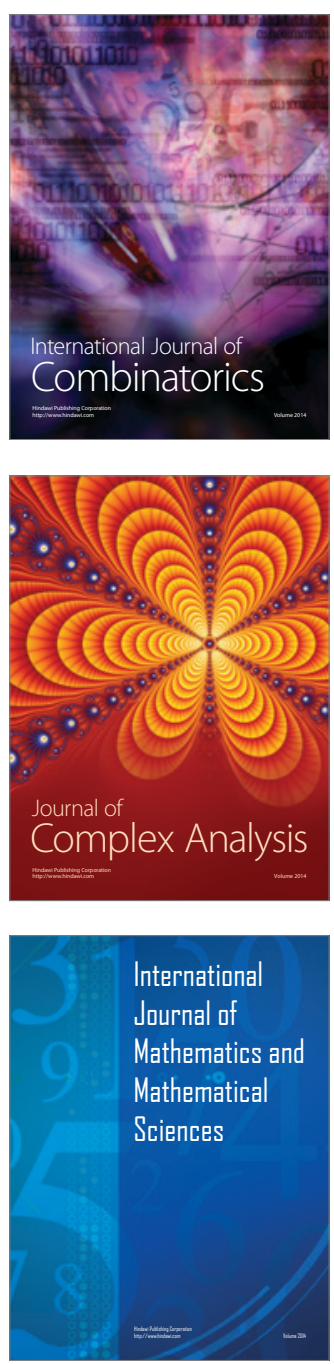
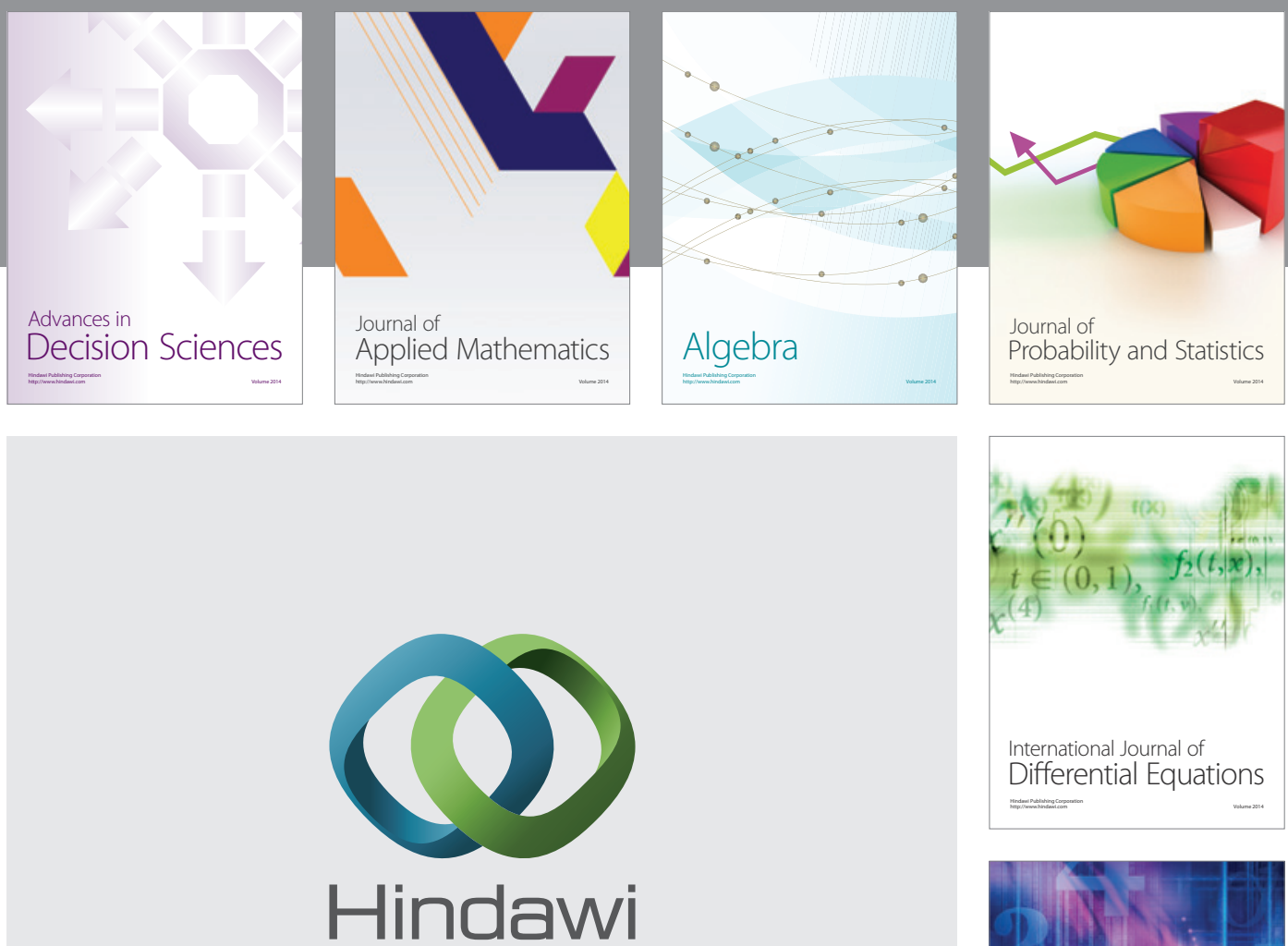

Submit your manuscripts at http://www.hindawi.com
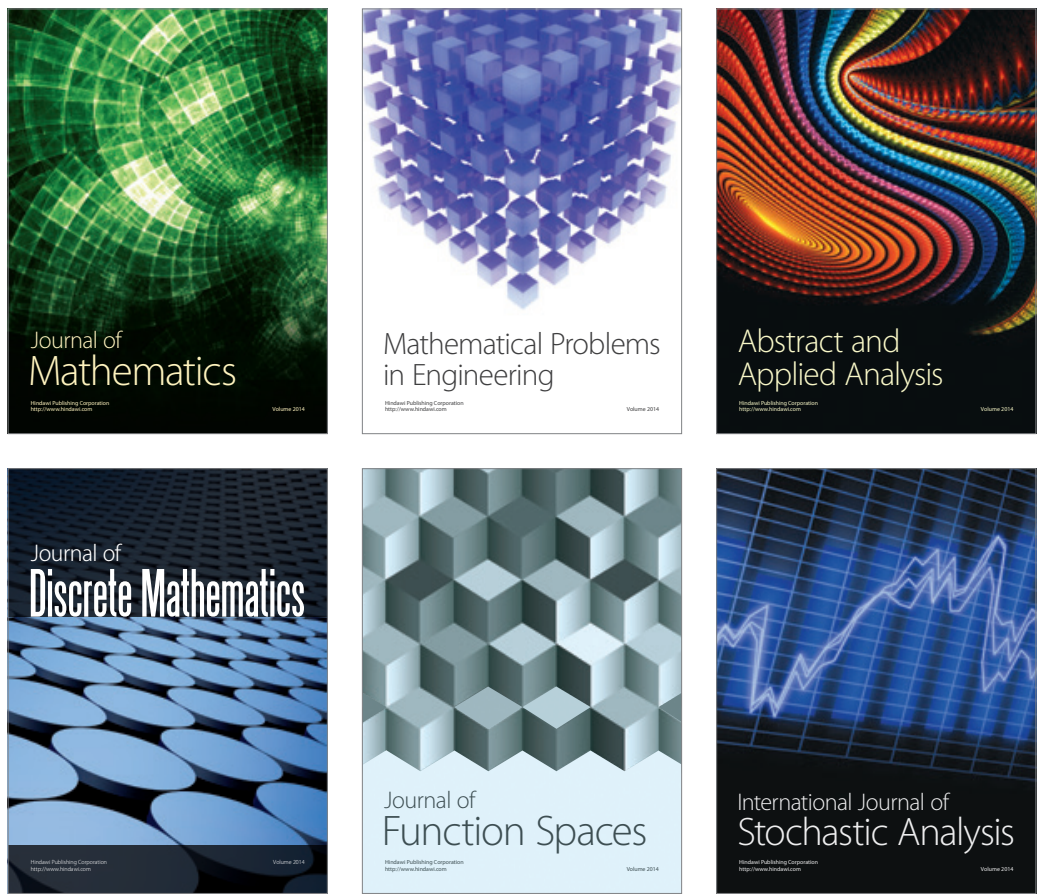

Journal of

Function Spaces

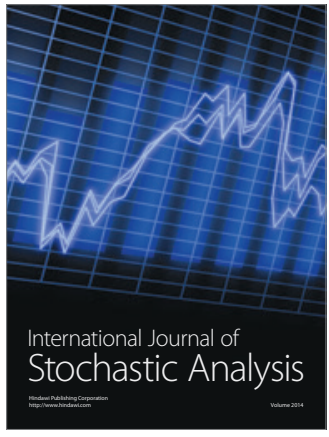

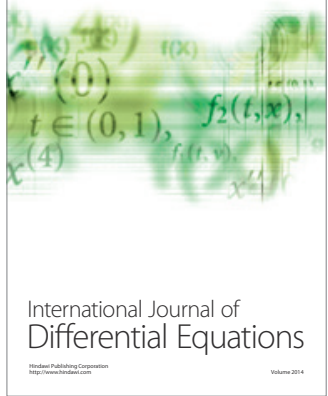
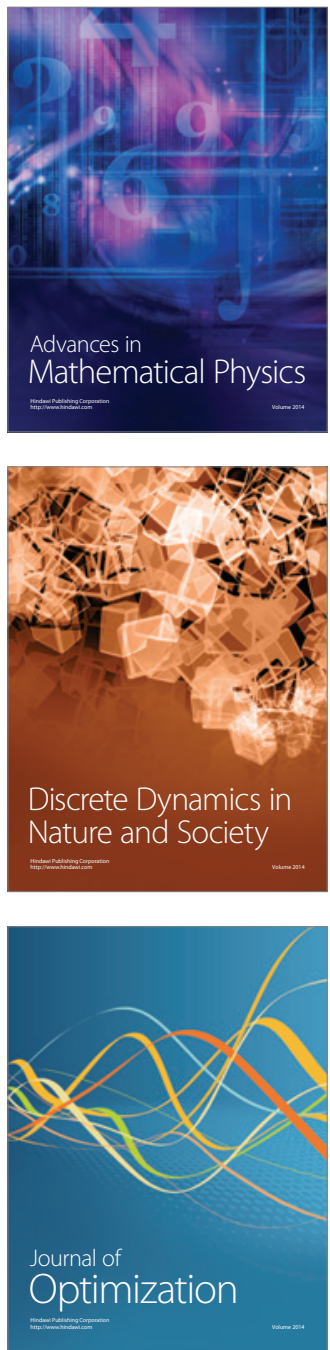